\title{
Factores asociados a insomnio en profesionales de salud de un hospital público de Trujillo-Perú
}

José Caballero-Alvarado 1,4,a; Francis Pino-Zavaleta ${ }^{2,4, b}$; Joshuan J. Barboza*3,4,c

\section{RESUMEN}

Objetivo: Identificar los factores asociados a insomnio en profesionales de salud de un hospital público de Trujillo, Perú. Materiales y métodos: Se aplicó la Encuesta de Insomnio de Atenas (EIA) a 220 profesionales de la salud. Se emplearon las pruebas de la ji al cuadrado y t de Student. El análisis multivariado fue utilizado para identificar las variables asociadas al insomnio.

Resultados: Se demostró que existe menos riesgo de insomnio en profesionales menores de 38 años (OR 0,77; IC 95\% $0,68-0,87 ; p<0,05)$. Existe mayor riesgo de presentar insomnio cuando el profesional de salud tiene una sobrecarga horaria en el trabajo (OR 4; IC 95\% 1,22-9,73; $p<0,05)$; si se trabaja en un turno nocturno (OR 3,12; IC 95\% 1,01-9,73; $p<0,05)$; si el trabajador labora por más de siete años en la institución (OR 1,50; IC 95\% 1,22-1,85; $p<0,001)$, y si el profesional de salud padece lumbalgia (OR 11,28; IC 95\% 3,61-35,28; $p<0,05$ ), esta última variable presenta el mayor valor de asociación.

Conclusiones: Nuestro estudio sugiere que los profesionales de la salud mayores de 38 años, con lumbalgia, sobrecarga laboral, trabajo nocturno y que trabajan más de siete años en la institución tienen mayor riesgo de riesgo de sufrir insomnio.

Palabras clave: Insomnio; Trastornos del inicio y del mantenimiento del sueño; Personal de salud (Fuente: DeCS BIREME).

\section{Factors associated with insomnia in health professionals of a public hospital in Trujillo, Peru}

\section{ABSTRACT}

Objective: To identify the factors associated with insomnia in health professionals of a public hospital in Trujillo, Peru. Materials and methods: The Athens Insomnia Scale (AIS) was administered to 220 health professionals. The chi-square test and Student's t-test were used. In addition, the multivariate analysis was performed to identify the variables associated with insomnia.

Results: It was demonstrated that there is less risk of insomnia in professionals under 38 years of age (OR $0.77 ; 95 \%$ $\mathrm{Cl} 0.68-0.87 ; p<0.05)$. There is more risk of insomnia in cases of work overload $(\mathrm{OR} 4 ; 95 \% \mathrm{Cl} 1.22-9.73 ; p<0.05)$, night shifts (OR 3.12; $95 \% \mathrm{Cl} 1.01-9.73 ; p<0.05)$, job tenure of more than 7 years in the hospital (OR $1.50 ; 95 \%$ $\mathrm{Cl} 1.22-1.85 ; p<0.001)$ and low back pain (OR 11.28; $95 \% \mathrm{Cl} 3.61-35.28 ; p<0.05)$, the latter being the variable with the highest association value.

Conclusions: Our study suggests that health professionals older than 38 years of age, with low back pain, under work overload, who work night shifts and with a job tenure of more than 7 years in the hospital are more at risk of suffering insomnia.

Keywords: Insomnia; Sleep initiation and maintenance disorders; Health personnel (Source: MeSH NLM).

1 Universidad Privada Antenor Orrego, Escuela de Medicina Humana. Trujillo, Perú.

2 Universidad Nacional de Trujillo, Sociedad Científica de Estudiantes de Medicina (SOCEMUNT).Trujillo, Perú.

3 Universidad Señor de Sipán. Chiclayo, Perú.

4 Tau-Relaped Group.

a Médico Cirujano, Doctor en Investigación Clínica.

b Estudiante de Medicina.

c Magister en Ciencias de la Investigación Clínica.

*Autor corresponsal. 


\section{INTRODUCCIÓN}

El insomnio es la imposibilidad para iniciar, mantener o conseguir una duración y calidad de sueño adecuadas para restaurar la energía y el estado de vigilia normal (1). La prevalencia del insomnio como síntoma de alguna enfermedad es elevada ${ }^{(2)}$. Se estima que un $50 \%$ de los adultos sufren insomnio en algún momento de la vida y que un 25 - $35 \%$ ha padecido insomnio ocasional o transitorio acompañando al estrés en situaciones vitales ${ }^{(3,4)}$.

Diversos factores se asocian a insomnio ${ }^{(5)}$, entre ellos, el sexo, la edad, el estado civil, la ocupación, la sobrecarga horaria y enfermedades como hipertensión arterial, diabetes mellitus, hipotiroidismo o lumbalgia ${ }^{(4,6,7)}$. En los Estados Unidos de América, el insomnio constituye el trastorno del sueño más frecuente; se estima que $76 \%$ de los afectados lo padece ${ }^{(8,9)}$. En España, un 20,8\% presenta al menos un síntoma relacionado con el insomnio tres o más veces a la semana ${ }^{(10)}$. Sin embargo, son pocos los estudios que se han realizado sobre calidad del sueño en el Perú, a pesar de su importancia.

Los profesionales de la salud tienen privación del sueño debido a sus horarios de trabajo o estudio ${ }^{(11)}$; por lo tanto, esto podría conducir a la fatiga y/o somnolencia, y esto repercute en el proceso del aprendizaje y desempeño laboral ${ }^{(8)}$. El trabajo de los profesionales de la salud en hospitales debe ser ininterrumpido, lo que les obliga a realizar un trabajo por turnos ${ }^{(12)}$; sin embargo, el trabajo es de alta presión, lo que puede ocasionar anomalías circadianas como el insomnio, esta situación puede influir en la calidad de atención que reciben los pacientes $y$, así mismo, este desorden del sueño puede tener repercusiones en la salud de los profesionales ${ }^{(10,11)}$.

Es importante remarcar que existen diferencias entre las condiciones de trabajo, sistemas de salud, soporte laboral entre otras condiciones, que varían entre regiones o países ${ }^{(13)}$, en función a esto, nos propusimos investigar los factores asociados a insomnio en profesionales de la salud de un hospital público y con esta información poder implementar estrategias de soporte para ellos.

\section{MATERIAL Y MÉTODOS}

\section{Diseño y población de estudio}

Estudio de tipo observacional analítico de corte transversal. La población de estudio estuvo conformada por 554 profesionales de salud del Hospital Belén (en Trujillo, al norte del Perú), durante el período comprendido entre mayo y julio de 2018.

\section{Variables y mediciones}

El tamaño muestral se obtuvo mediante la fórmula del tamaño de muestra para una proporción y se obtuvo un total de 220 individuos. Para ello se utilizó un valor de $p=0,05$ que fue una prevalencia de $7 \%$. En la investigación se incluyeron a los profesionales de la salud (médicos y enfermeros) que aceptaron participar en el estudio. Se excluyeron a los trabajadores que no estaban laborando en el hospital durante la obtención de datos, que se retiraron durante el desarrollo de la investigación y a los que tenían un diagnóstico de estrés postraumático o con otros trastornos mentales, y también las encuestas con datos incompletos. Se realizó un muestreo no probabilístico por conveniencia. Las visitas se realizaron a los servicios asistenciales de Medicina, Cirugía, Pediatría y Ginecología y Obstetricia entre las 12 a las 14 horas, tiempo en el que el trabajo es menor y, generalmente, se realizan actividades administrativas. La variable de interés fue el insomnio, que se definió como la dificultad para iniciar o mantener el sueño ${ }^{(2)}$. Para determinar la presencia o ausencia de insomnio se utilizó la Escala de Insomnio de Atenas (EIA). La EIA es un instrumento psicométrico autoadministrado que consta de ocho elementos. Son tres criterios para el diagnóstico de insomnio agudo según la EIA. Los cinco primeros ítems de EIA (evaluación de la dificultad para inducir el sueño, los despertares durante la noche, el despertar temprano en la mañana, el tiempo total de sueño y la calidad general del sueño) corresponden al criterio A para el diagnóstico del insomnio según la $\mathrm{CIE}-10$, mientras que los requisitos de una frecuencia mínima (al menos tres veces por semana) y la duración (1 mes) de cualquier queja corresponden al criterio $\mathrm{B}$ de la CIE-10. Los requisitos de la CIE10 como marcada angustia causada por el problema de sueño y/o interferencia con las actividades ordinarias de la vida diaria (criterio C) son de naturaleza estrictamente subjetiva de las opciones de respuesta para cada ítem de la escala, así como a través del contenido de los tres últimos ítems relativos a las consecuencias del insomnio al día siguiente (problemas con sensación de bienestar, funcionamiento y somnolencia durante el día). Cada elemento del EIA puede tener una calificación de $0 \pm 3$, (en que 0 corresponde a "ningún problema en absoluto" y 3 a "problema muy grave"). Se pidió a los encuestados que dieran una calificación positiva si han experimentado la dificultad para dormir descrita en cada ítem al menos tres veces a la semana durante el último mes o algún otro período de tiempo, cuya duración depende del propósito de un estudio determinado ${ }^{(14)}$. Las variables analizadas fueron categorizadas como sociodemográficas, laborales y clínicas. Se consideraron edad, sexo, tipo de profesión sanitaria, hipertensión arterial, diabetes mellitus, hipotiroidismo, lumbalgia, consumo habitual de alcohol, hábito de fumar tabaco, sobrecarga horaria, turnos nocturnos, años de graduado y años de labor en la institución. Tales datos fueron obtenidos a través de preguntas elaboradas por el equipo investigador.

\section{Análisis estadístico}

Se realizó un análisis descriptivo de las características 
sociodemográficas, laborales y clínicas de los participantes. Las variables continuas fueron analizadas utilizando medidas de resumen como media y desviación estándar, o mediana y rango según su distribución normal y no paramétrica, respectivamente. Las variables categóricas se describieron mediante frecuencias absolutas y relativas. Se utilizó la prueba de ji al cuadrado de Pearson para comparar variables cualitativas y la prueba $t$ de Student para comparar la media de variables cuantitativas. Además, se realizó un análisis multivariado con la regresión logística para identificar las variables exposición asociados al insomnio, para obtener el OR ajustado mediante variables de confusión y su IC $95 \%$. Se estableció el grado de significancia estadística, con un valor de $p<0,05$. Para el análisis estadístico se utilizó el software R studio ( $\vee 1.1 .463)$.

\section{Consideraciones éticas}

Hemos asegurado la confidencialidad de la información de cada entrevistado, asignando solo códigos a cada entrevista, con el consentimiento informado previo. Se han respetado los principios éticos de la Declaración de Helsinki, así como el informe de la investigación.

\section{RESULTADOS}

La muestra estuvo conformada por 220 profesionales de la salud, de los cuales 32 (17,00 \%) padece de insomnio. La tabla 1 muestra el análisis bivariado de las características sociodemográficas, laborales y clínicas asociadas a insomnio en los profesionales de la salud. Las características sociodemográficas significativamente asociadas a insomnio son el consumo habitual de alcohol, así como el hábito de fumar. Los factores laborales asociados a insomnio fueron la sobrecarga horaria y los turnos nocturnos. Finalmente, los factores clínicos asociados a insomnio, se observa que la hipertensión arterial, la diabetes mellitus, el hipotiroidismo, así como la lumbalgia, tienen un nivel de asociación significativo en el insomnio en profesionales de la salud.

Tabla 1. Análisis bivariado de las características sociodemográficas, laborales y clínicas asociadas a insomnio en profesionales de salud del Hospital Belén, Trujillo (mayo - julio 2018)

Variables Insomnio $n(\%) \quad$ OR* (IC 95\%) ** Valor de $p$

\begin{tabular}{|c|c|c|c|c|}
\hline & Sí $(n=32)$ & No $(n=188)$ & & \\
\hline Edad (media en años) & $38,28 \pm 7,12$ & $40,73 \pm 7,21$ & NA & 0,07 \\
\hline \multicolumn{5}{|l|}{ Sexo } \\
\hline Masculino & $20(62,00 \%)$ & $118(63,00 \%)$ & $1,01(0,47-2,19)$ & 0,98 \\
\hline Femenino & $12(38,00 \%)$ & $70(37,00 \%)$ & & \\
\hline \multicolumn{5}{|l|}{ Consumo habitual de alcohol } \\
\hline Sí & $5(16,00 \%)$ & $3(2,00 \%)$ & $11,42(2,58-50,53)$ & $<0,001$ \\
\hline No & $27(84,00 \%)$ & $185(98,00 \%)$ & & \\
\hline \multicolumn{5}{|l|}{ Hábito de fumar tabaco } \\
\hline Sí & $4(13,00 \%)$ & $3(2,00 \%)$ & $8,81(1,87-41,46)$ & $<0,001$ \\
\hline No & $28(88,00 \%)$ & $185(98,00 \%)$ & & \\
\hline Años de graduado & $9,75 \pm 3,22$ & $8,95 \pm 5,65$ & NA & 0,44 \\
\hline Años de labor en la institución & $7,34 \pm 2,98$ & $6,29 \pm 4,28$ & NA & 0,18 \\
\hline \multicolumn{5}{|l|}{ Tipo de personal } \\
\hline Médico & $12(38,00 \%)$ & $81(43,00 \%)$ & $0,79(0,37-1,72)$ & 0,55 \\
\hline No médico & $20(63,00 \%)$ & $107(57,00 \%)$ & & \\
\hline \multicolumn{5}{|l|}{ Sobrecarga horaria } \\
\hline Sí & $8(25,00 \%)$ & $17(9,00 \%)$ & $3,35(1,31-8,61)$ & 0,01 \\
\hline No & $24(75,00 \%)$ & $171(91,00 \%)$ & & \\
\hline
\end{tabular}


Turnos nocturnos

\begin{tabular}{|c|c|c|c|c|}
\hline Sí & $10(31,00 \%)$ & $23(12,00 \%)$ & $3,26(1,37-7,75)$ & 0,01 \\
\hline No & $22(69,00 \%)$ & $165(88,00 \%)$ & & \\
\hline \multicolumn{5}{|c|}{ Hipertensión arterial } \\
\hline Sí & $6(19,00 \%)$ & $2(1,00 \%)$ & $21,46(4,11-111,98)$ & $<0,001$ \\
\hline No & $26(81,00 \%)$ & $186(99,00 \%)$ & & \\
\hline \multicolumn{5}{|c|}{ Diabetes mellitus } \\
\hline Sí & $3(9,00 \%)$ & $3(2,00 \%)$ & $6,38(1,23-33,13)$ & 0,01 \\
\hline No & $29(91,00 \%)$ & $30(94,00 \%)$ & & \\
\hline \multicolumn{5}{|c|}{ Hipotiroidismo } \\
\hline Sí & $2(6,00 \%)$ & $1(1,00 \%)$ & $12,47(1,10-141,78)$ & 0,01 \\
\hline No & $30(94,00 \%)$ & $187(99,00 \%)$ & & \\
\hline \multicolumn{5}{|c|}{ Lumbalgia } \\
\hline Sí & $13(41,00 \%)$ & $9(5,00 \%)$ & $13,61(5,15-35,99)$ & $<0,001$ \\
\hline No & $19(59,00 \%)$ & $179(95,00 \%)$ & & \\
\hline
\end{tabular}

* OR: Odds ratio

${ }^{* *}$ IC 95\%: Intervalo de confianza al 95\%

En la tabla 2 se muestra el análisis multivariado con OR ajustado (ORa) de los factores asociados a insomnio en profesionales de la salud. Se observa que hay menor probabilidad de insomnio en profesionales con edad menor a 38 años (ORa de 0,$77 ;$ IC $95 \%: 0,68-0,87 ; p<0,001$ ). Por otro lado, se observa hay más probabilidad de que los profesionales sufran insomnio cuando está asociado a lumbalgia (OR de 11,28; IC 95\%: 3,61-35,28; $p<0,05)$, sobrecarga horaria en el trabajo (OR de 4; IC 95\%: 1,22-9,73; $p<0,05$ ), en los que realizan turnos nocturnos (OR de 3,12; IC $95 \%$ : 1,01-9,73; $p<0,05)$, y en aquellos que tienen más de siete años de labor en la institución (OR de 1,$50 ;$ IC $95 \%: 1,22-1,85 ; p<0,05)$.

Tabla 2. Análisis multivariado de los factores asociados a insomnio en personal profesional de salud del Hospital Belén, Trujillo (mayo - julio 2018)

\begin{tabular}{|c|c|c|c|c|c|c|}
\hline \multirow{2}{*}{ Variables } & \multirow{2}{*}{ Constante } & \multirow{2}{*}{ WALD † } & \multirow{2}{*}{ Valor de $p$} & \multirow{2}{*}{ ORa* } & \multicolumn{2}{|c|}{ IC $95 \%$ ** } \\
\hline & & & & & Inferior & Superior \\
\hline Edad $<38$ años & $-0,26$ & 16,72 & $<0,001$ & 0,77 & 0,68 & 0,87 \\
\hline Lumbalgia & 2,42 & 17,35 & $<0,001$ & 11,28 & 3,61 & 35,28 \\
\hline Sobrecarga horaria & 1,39 & 5,27 & 0,02 & 4,0 & 1,23 & 13,08 \\
\hline Turnos nocturnos & 1,14 & 3,88 & 0,05 & 3,13 & 1,01 & 9,73 \\
\hline Tiempo de laborar & 0,41 & 14,85 & 0,001 & 1,50 & 1,22 & 1,85 \\
\hline
\end{tabular}

* ORa: Odds ratio ajustado

**IC 95\%: Intervalo de confianza al 95\%

† WALD: Valor de la prueba de Wald para reconocer los valores de verosimilitud de las variables en una regresión logística 


\section{DISCUSIÓN}

El interés por abordar el insomnio y sus consecuencias son una arista adicional en la salud pública debido al impacto y las repercusiones que tienen en la calidad de vida de las personas que lo padecen ${ }^{(15)}$. Los problemas de sueño se han asociado con un mayor riesgo de alteraciones de la salud mental, especialmente, con el desarrollo de trastornos depresivos ${ }^{(16)}$. Igualmente, se han encontrado relación con el ausentismo laboral, con los cambios de humor y los problemas del comportamiento, así como con la vulnerabilidad al consumo de drogas y alcohol en los profesionales de salud ${ }^{(17,18)}$.

Nuestro estudio determinó que los profesionales de la salud con una edad menor a 38 años tienen menos riesgo de padecer de insomnio. Fernandes-Portela et al. (19) determinaron que la prevalencia de síntomas de insomnio fue de $34,3 \%$ y que fue mayor en enfermeras con edades a partir de 35 años. Podemos observar que la edad influye en el riesgo de insomnio, de esto se infiere que aquellos profesionales más jóvenes tienen funciones asistenciales distintas a la de profesionales de mayor edad que, además, tienen mayor destreza y vitalidad.

La edad promedio del ingreso a la labor hospitalaria es 24 años, los profesionales menores de 38 años tienen, al menos, 15 años de labor asistencial en centros clínicos. Esto indica que se puede estudiar el insomnio en profesionales con un punto de corte mayor a esta edad, y así poder conocer el impacto en los profesionales con mayor tiempo en ejercicio de su profesión.

Los profesionales de salud con mayor tiempo laboral están en mayor riesgo de sufrir insomnio ${ }^{(20)}$, lo cual puede afectar su rendimiento laboral y tener impacto negativo en quienes reciben atención, es decir, los pacientes. En este sentido, Chang et al. (21) hallaron un mayor riesgo de insomnio en las enfermeras de mayor edad. Estos resultados indican que la edad avanzada está asociada con mayor prevalencia de insomnio, esto puede deberse a que los profesionales de la salud con rangos de edad mayores pueden ser más sensibles a la fase circadiana y puede tener más dificultad para dormir lo suficiente.

Aunque no se puede determinar exactamente los rangos de edad para determinar cuál grupo tiene mayor riesgo, existen estudios que analizan la presencia de síntomas en otros grupos etarios. Así, Rueda Sánchez et al. ${ }^{(13)}$ encontraron que la prevalencia de insomnio es mayor en el rango de edades de 46 a 65 años. En cuanto a la relación de turnos nocturnos con el insomnio nuestro estudio determinó que los profesionales de la salud con turnos nocturnos tienen más riesgo de padecer insomnio. Thun et al. (2) encontraron que los profesionales de la salud que abandonaron el trabajo nocturno tuvieron una disminución de los síntomas de insomnio con el tiempo, en comparación con las que se quedaron en dicho horario. Este estudio permite inferir la implicancia del desvelo durante la guardia nocturna y el posterior insomnio al que se ven sometidos los profesionales de salud.

El desvelo continuo y falta de recuperación de la energía y el sueño de calidad son los principales desencadenantes de insomnio en los profesionales de salud, aunado a todos los factores personales y clínicos ${ }^{(12,19,22)}$. El trabajo nocturno genera más que cansancio, agotamiento, puesto que es imposible realizar periodos cortos de sueño e intentar compensar la fatiga que genera el permanecer despierto varias horas durante la noche; además, se agregan la preocupación por la atención y la vigilancia permanente que son inherentes al trabajo intrahospitalario (11). Son pocos los estudios que han considerado el impacto del trabajo nocturno, por la "normalidad" que obliga la responsabilidad laboral (23). En este sentido, Flo et al. (14) reportaron síntomas indicativos de insomnio-somnolencia en el $44,2 \%$ de las enfermeras que trabajaban en turno de la noche, y en el $23,6 \%$ de las que no trabajaron en turnos de noche.

Esta investigación determinó que los profesionales de la salud con sobrecarga horaria, es decir, mayor cantidad de horas adicionales a la jornada laboral, tienen más riesgo de presentar insomnio. Virtanen et al. (24), concluyeron que a mayor número de horas de trabajo, existe un riesgo mayor de padecer enfermedades cognitivas relacionadas al cansancio y Díaz Cabezas et al. ${ }^{(25)}$ reportaron que a mayor carga horaria existe mayor riesgo de trastorno de sueño. La sobrecarga horaria se relaciona con el cansancio físico y mental, ambos son factores desencadenantes de cuadros de estrés y ansiedad, que pueden ocasionar insomnio.

Finalmente, nuestro estudio determinó que los profesionales de la salud que padecen de lumbalgia tienen más riesgo de presentar insomnio. Si consideramos que la mayoría de los profesionales de salud desarrollan su labor asistencial en constante movimiento, adoptar posturas ergonómicas muchas veces es secundario o ignorado, lo que conlleva a desarrollar lumbalgias que, finalmente, pueden afectar la calidad del sueño y generar insomnio.

De este modo, los profesionales de salud, pilar esencial de sus centros de trabajo, demandan una atención especial en cuanto a su propio cuidado. Es notorio que los profesionales más jóvenes tienen menor dificultad para el desarrollo asistencial de su trabajo, pero no están exentos de tener problemas relacionados a trastornos del sueño, mucho más si presentan otros factores de riesgo como los identificados en este trabajo. No se han realizado más estudios acerca de los factores asociados, así como tampoco se toman medidas para evaluar la calidad del sueño y el desempeño laboral, lo que es importante porque un profesional de 
salud en óptimas condiciones funcionales, va a rendir con mayor efectividad en su labor cotidiana.

Nuestra investigación presenta algunas limitaciones, una de ellas es el diseño del estudio que no permite tener la certeza de si los profesionales de salud tenían insomnio previamente; y después aparecieron condiciones como el lumbago, la carga laboral en los profesionales de salud que pueden variar, lo que estaría relacionado a presentar insomnio en grados distintos. Por otro lado, es probable que haya otras variables que no fueron estudiadas y que influyen en la presencia de insomnio, por ejemplo, la ansiedad, depresión, problemas médico-legales, condiciones que son frecuentes en hospitales públicos. Este estudio es relevante por el abordaje de una enfermedad poco tratada en los profesionales de salud, puesto que se ha estigmatizado el insomnio como consecuencia únicamente del esfuerzo, y no se han analizado sus implicancias fisiológicas, laborales y personales.

Es recomendable realizar estudios futuros que incluyan otras variables, e incluir otro tipo de hospitales, como los de la seguridad social, este hecho permitirá extrapolar los resultados a la gran población de trabajadores de la salud.

Se concluye que hay menor riesgo de insomnio en profesionales de salud menores de 38 años. Por otro lado, padecer lumbalgia, tener sobrecarga laboral, trabajar en turnos nocturnos y laborar más de siete años en la institución son factores asociados para presentar insomnio en los profesionales de salud.

Exención de responsabilidad: Los autores confirman que el contenido del presente manuscrito, es producto del escrutinio aplicado al participante y responsabilidad estrictamente de los profesionales involucrados en la publicación de este estudio.

Contribución de los autores: José Caballero-Alvarado ha participado en la concepción y diseño del artículo, recolección de resultados, análisis e interpretación de datos, aporte de pacientes o material de estudio, y en la redacción, revisión crítica del artículo y aprobación de la versión final del trabajo. Joshuan J. Barboza-Meca ha participado en la concepción y diseño del artículo; análisis e interpretación de datos, aporte de pacientes o material de estudio, y en la redacción, revisión crítica del artículo y aprobación de la versión final del trabajo. Francis Pino Zavaleta ha participado en la revisión crítica del articulo y aprobación de la versión final.

Fuente de financiamiento: El artículo ha sido financiado por los autores.

Conflictos de interés: Los autores declaran no tener ningún conflicto de interés.

\section{REFERENCIAS BIBLIOGRÁFICAS}

1. Tsai K, Lee T-Y, Chung M-H. Insomnia in female nurses: a nationwide retrospective study. Int J Occup Saf Ergon. 2017; 23(1): 127-32.

2. Thun E, Bjorvatn B, Akerstedt T, Moen BE, Waage S, Molde H, et al. Trajectories of sleepiness and insomnia symptoms in Norwegian nurses with and without night work and rotational work. Chronobiol Int. 2016; 33(5): 480-9.

3. Vela-Bueno A, Iceta $M$, Fernández C. Prevalencia de los trastornos del sueño en la ciudad de Madrid. Gac San. 1999; 13(6): 441-8.

4. Sarrais F, De Castro Manglano P. El insomnio. Anales Sis San Navarra. 2007; 30(Supl. 1): 121-34.

5. Amelot A, Cretolle C, de Saint Denis T, Sarnacki S, Catala M, Zerah M. Spinal dysraphism as a new entity in V.A.C.TE.R.L syndrome, resulting in a novel acronym V.A.C.TE.R.L.S. Eur J Pediatr. 2020; 179(7): 1121-9.

6. Barrientes KM, Arango SG, Bertel FC, Marriaga A, Arias AC. Prevalencia de insomnio y sonmnolencia en estudiantes de medicina pertenecientes a una institución educativa de Medellín. Arch Med (Col). 2014; 14(1): 92-9.

7. Shen S-H, Yen M, Yang S-L, Lee C-Y. Insomnia, anxiety, and heart rate variability among nurses working different shift systems in Taiwan. Nurs Health Sci. 2016; 18(2): 223-9.

8. Oyane NMF, Pallesen S, Moen BE, Akerstedt T, Bjorvatn B. Associations between night work and anxiety, depression, insomnia, sleepiness and fatigue in a sample of Norwegian nurses. PLoS One. 2013; 8(8): e70228.

9. García Gascón Á, Querts Méndez O, Hierrezuelo Izquierdo EJ, Quesada Vidal S. Algunos aspectos psicosociales del insomnio en estudiantes de primer año de medicina. MEDISAN. 2015; 19(1): 56-60.

10. Torrens I, Ortuño M, Guerra JI, Esteva M, Lorente P. Actitudes ante el insomnio de los médicos de AP de Mallorca. Atención Primaria. 2016; 48(6): 374-82.

11. Bonet-Porqueras R, Moliné-Pallarés AA, Olona-Cabases M, Gil-Mateu $E$, Bonet-Notario $P$, Les-Morell $E$, et al. Turno nocturno: un factor de riesgo en la salud y calidad de vida del personal de enfermería. Enferm Clin. 2009; 19(2): 76-82.

12. Geiger-Brown J, Rogers VE, Trinkoff AM, Kane RL, Bausell RB, Scharf SM. Sleep, sleepiness, fatigue, and performance of 12-hour-shift nurses. Chronobiol Int. 2012; 29(2): 211-9.

13. Rueda Sánchez M, Díaz Martínez LA, Osuna Suarez E. Definición, prevalencia y factores de riesgo de insomnio en la población general. Rev Fac Med. 2008; 56(3): 222-34.

14. Flo E, Pallesen S, Mageroy N, Moen BE, Gronli J, Nordhus IH, et al. Shift work disorder in nurses-assessment, prevalence and related health problems. PLoS One. 2012; 7(4): e33981.

15. Barrenechea Loo MB, Gomez Zeballos C, Huaira Peña AJ, Pregúntegui Loayza I, Aguirre Gonzales M, de Castro Mujica RJ. Calidad de sueño y excesiva somnolencia diurna en estudiantes del tercer y cuarto año de Medicina. CIMEL. 2010; 15(2).

16. Bjorvatn B, Pallesen S, Moen BE, Waage S, Kristoffersen ES. Migraine, tension-type headache and medication-overuse headache in a large population of shift working nurses: a cross-sectional study in Norway. BMJ Open. 2018; 8(11): e022403.

17. Rodríguez-Muñoz A, Moreno-Jiménez B, Fernández-Mendoza JJ, Olavarrieta-Bernardino S, De la Cruz-Troca JJ, Vela-Bueno A. Insomnio y calidad del sueño en médicos de atención primaria: una perspectiva de género. Rev Neurol. 2008; 47(3): 119-223.

18. Furihata R, Saitoh K, Otsuki R, Murata S, Suzuki M, Jike M, et al. Association between reduced serum BDNF levels and insomnia with short sleep duration among female hospital nurses. Sleep Med. 2020; 68: $167-72$. 
Factores asociados a insomnio en profesionales de salud de un hospital público de Trujillo-Perú

19. Fernandes-Portela L, Kroning Luna C, Rotenberg L, Silva-Costa A, Toivanen S, Araújo T, et al. Job strain and self-reported insomnia symptoms among nurses: what about the influence of emotional demands and social support?. BioMed Res Int. 2015; 2015(1): 1-9.

20. Huang $\mathrm{CL}$, Wu MP, Ho CH, Wang JJ. Risks of treated anxiety, depression, and insomnia among nurses: a nationwide longitudinal cohort study. PLOS ONE. 2018; 13(9): e0204224.

21. Chang $Y$, Lam C, Chung $M-H$. Influence of new hire transition workload on insomnia in nurses. Int J Nurs Pract. 2018; 24(5): e12666.

22. Ohayon MM. Epidemiology of insomnia: what we know and what we still need to learn. Sleep Med Rev. 2002; 6(2): 97-111.

23. Avidan AY, Neubauer DN. Chronic insomnia disorder. Continuum (Minneap Minn). 2017; 23(4): 1064-92.

24. Virtanen M, Singh-Manoux A, Ferrie JE, Gimeno D, Marmot MG, Elovainio $M$, et al. Long working hours and cognitive function: the Whitehall II Study. Am J Epidemiol. 2009; 169(5): 596-605.

25. Díaz Cabezas R, Ruano Restrepo MI, Chacón Cardona A. Estudio de trastornos de sueño en Caldas, Colombia (SUECA). Acta Med Colomb. 2009; 34(2): 66-72.

\section{Correspondencia:}

Joshuan J. Barboza-Meca

Dirección: Calle Juan del Corral 937. Trujillo, La Libertad. Teléfono:+ 51937285479

Correo electrónico: jbarbozameca@relaped.com

Recibido: 23 de abril de 2020

Evaluado: 24 de agosto de 2020

Aprobado: 01 de setiembre de 2020

(c) La revista. Publicado por Universidad de San Martín de Porres, Perú.

(cc) Br Licencia de Creative Commons Artículo en acceso abierto bajo términos de Licencia Creative Commons Atribución 4.0 Internacional. (http://creativecommons.org/licenses/by/4.0/)

\section{ORCID iDs}

José Caballero-Alvarado

Joshuan J. Barboza-Meca

Francis Pino - Zavaleta
(1) https: / / orcid.org/0000-0001-8297-6901
간 https: / / orcid.org/0000-0002-2896-1407
(1) https: / / orcid.org/0000-0003-1474-197X 